\title{
Determination of thermal parameters of one-dimensional nanostructures through a thermal transient method
}

\author{
A. Arriagada $\cdot$ E. T. Yu $\cdot$ P. R. Bandaru
}

Received: 3 November 2008/ Accepted: 26 June 2009/Published online: 6 August 2009

(C) The Author(s) 2009. This article is published with open access at Springerlink.com

\begin{abstract}
We present an improved methodology for a thermal transient method enabling simultaneous measurement of thermal conductivity and specific heat of nanoscale structures with one-dimensional heat flow. The temporal response of a sample to finite duration heat pulse inputs for both short $(1 \mathrm{~ns})$ and long $(5 \mu \mathrm{s})$ pulses is analyzed and exploited to deduce the thermal properties. Excellent agreement has been obtained between the recovered physical parameters and computational simulations through choosing an optimized pulse width.
\end{abstract}

Keywords Thermal conductivity - Nanoscale ·

Simulation

Accurate thermal characterization of low-dimensional structures is necessary both for fundamental understanding of heat transport at the nano-/macro-scale and for practical purposes such as determining the figure of merit of thermoelectric materials. However, the steady-state methods typically employed suffer from difficulties with thermal gradients and also require separate experiments to determine individual values of thermal conductivity $(k)$, specific heat $(C)$, and thermal diffusivity $(D)$. Transient techniques can be exploited to circumvent the above difficulties $[1,2]$.

\footnotetext{
A. Arriagada $(\bowtie) \cdot$ E. T. Yu

Department of Electrical and Computer Engineering, University

of California at San Diego, La Jolla, CA 92093-0407, USA

e-mail: aarriaga@ucsd.edu

P. R. Bandaru

Materials Science Program, Mechanical Engineering

Department, University of California at San Diego, La Jolla,

CA 92093-0411, USA
}

An early experiment in this regard entailed monitoring heat pulse propagation at a particular location on the sample and computation of the statistical moments of the temperature ( $T$ ) versus time $(t)$ curve to determine $k$ and $C$ [3]. In this case, the dispersion of a heat pulse as it travels down a sample as shown schematically in Fig. 1 can be quantified through the computation of its moments and related to the thermal parameters appearing in the solution of the corresponding heat equation (obtained through Laplace transform techniques). The moments, $f_{n}$, have been defined as [4]

$f_{n}=\int_{0}^{\infty} \Delta T(x, t) t^{n} d t$ for $-\infty<n<\infty$,

where $\Delta T(x, t)$ is the measured temperature change as a function of time $(t)$ at a point $(x)$ along the sample in response to a heat pulse, and $n$ refers to the order of the moment. Through a differentiation theorem [5], it can be shown that these moments can also be expanded in terms of the Laplace transform of the temperature profile, $\Delta T(x, s)$, through

$f_{n}=(-1)^{n} \lim _{s \rightarrow 0} \frac{d^{n} \Delta T(x, s)}{d s^{n}}$ for $n \geq 0$,

where $s$ is the complex variable introduced in the Laplace transform. In Eq. 2, $\Delta T(x, s)$ can be taken to be the Laplace transform of the solution for the heat conduction equation. The moments method then relates the time-weighted integrals of $\Delta T(x, t)$ to derivatives of $\Delta T(x, s)$ through Eq. 2 to allow $k$ and $C$ to be extracted, and has the advantage that it can be modified to account for the influence of auxiliary thermal sources and sinks (i.e. heaters, thermometers, substrate/supporting structures, etc.) [6-8].

While the above formulation in its original form using $\delta$-function like pulses is adaptable for nanoscale structures with one-dimensional heat flow, its application has been 

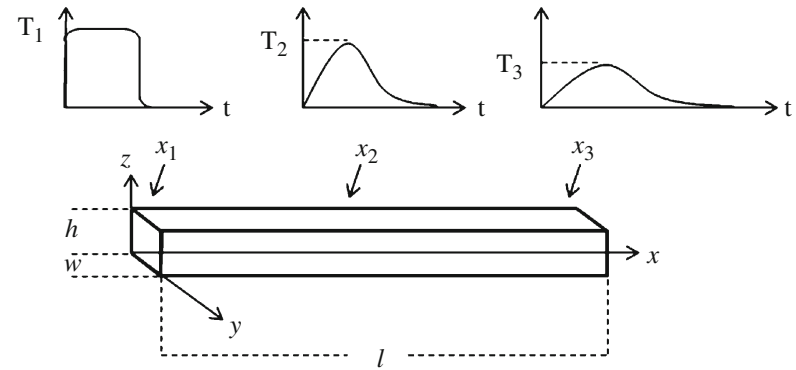

Fig. 1 Schematic of heat pulse propagation (top) along a rectangular nanowire (bottom). The temperature $(T)$ vs. time $(t)$ plots show the dispersion of an input (at $x=0$ ) finite duration heat pulse as it diffuses, from left to right, along the wire

hindered by the fact that large geometric aspect ratios (inherent to nanotubes, nanowires, and thin films) and small energies $(\mathrm{fJ}-\mathrm{pJ})$ yield temperature changes $(\Delta T)$ that are very difficult to measure. Larger temperature changes through resistive heating can be achieved by increasing the heater power. However a heater cross-sectional area large enough to avoid electromigration [9] can also lead to undesirable thermal parasitics that complicate analyses. Alternately, increasing the applied current duration can cause deviations in the experimental temperature profiles resulting in substantial errors in the computation of the moments. In this letter, we reconsider the form of the input energy for a nanowire (assuming a rectangular cross-sectional area) to provide a new experimental formulation for $k$ and $C$, and then demonstrate the recovery of these thermal parameters through simulations and improved computational methods.

We assume a one-dimensional heat conduction equation approximation which is valid when the sample is taken to be a wire with a very large aspect ratio (e.g. nanowire) with the appropriate boundary conditions. The heat pulse and heat sink are applied uniformly across the ends of the wire inducing isothermal cross sections normal to the wire axis. Therefore, the heat conduction along an isolated square wire (Fig. 1) of cross-sectional area $A(=w h)$ and length $l$, where $l \gg w, h$, is described by

$\frac{\partial^{2} T}{\partial x^{2}}=R^{\prime} C^{\prime} \frac{\partial T}{\partial t}$

where $R^{\prime}=1 / k A$ is the thermal resistivity and $C^{\prime}=C \rho A$ is the specific heat (both per unit length) and $\rho$ is the density of the wire material. We then apply the following boundary and initial conditions at ambient temperature $T_{0}$ :

$T(x=l, t)=T(x=0, t)=T_{0}$,

$T(x, t=0)=T_{0} \quad$ for $\quad x \neq 0$,

$P(x=0, t)=P_{0}([u(t)-u(t-\tau)]) \quad$ for all $t$,

where $P_{0}$ is the input power magnitude, $u(t)$ is a unit step function, and $P(x=0, t)$ defines a heat pulse of duration $\tau$ with total energy $Q=P_{0} \tau$. Consideration of very small durations $(\tau \rightarrow 0)$ will lead to the original pulse-based moments method [10-12], while a long duration pulse $(\tau \rightarrow \infty)$ would imply steady-state heat conduction. In the following, we work with finite duration input pulses, resulting in responses exhibiting both transient and steadystate attributes and enabling the determination of both $k$ and $C$ from a single experimental measurement.

The problem is modeled as a thermal analog to the electrical transmission line problem and its solution determined using Laplace transform techniques. For the specified boundary and initial conditions, we have derived the change in temperature along the wire, for a given crosssectional area, to be

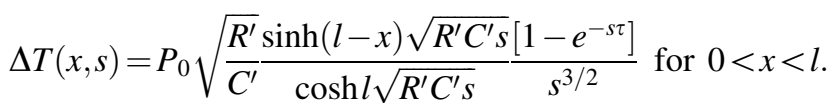

The moments are then evaluated using Eq. 2 from the solution of an equivalent transmission line model for the wire, given by Eq. 7, and related to a known temperature profile, $\Delta T(x, t)$, through Eq. 1. At least two of the moments must be calculated for determining the two desired thermal parameters, $k$ and $C$. For $n=0,1$ and 2, we find that

$$
\begin{aligned}
f_{n=0}= & \int_{0}^{\infty} \Delta T(x, t) d t=P_{0} R^{\prime} \tau(l-x) \\
f_{n=1}= & \int_{0}^{\infty} \Delta T(x, t) t d t=\frac{1}{6} P_{0} R^{\prime 2} C^{\prime} \tau(l-x)\left(2 l^{2}+2 l x-x^{2}\right) \\
& +\frac{1}{2} P_{0} R^{\prime} \tau^{2}(l-x), \\
f_{n=2}= & \int_{0}^{\infty} \Delta T(x, t) t^{2} d t=\frac{1}{60} P_{0} R^{\prime 3} C^{\prime 2} \tau(l-x) \\
& \times\left(-4 l^{2}-2 l x+x^{2}\right)^{2}+\frac{1}{6} P_{0} R^{\prime 2} C^{\prime} \tau^{2}(l-x) \\
& \times\left(2 l^{3}-3 l x^{2}+x^{3}\right)+\frac{1}{3} P_{0} R^{\prime} \tau^{3}(l-x)
\end{aligned}
$$

If $\Delta T(x, t)$ is determined experimentally, Eqs. 8-10 can be evaluated in units of $[K s],\left[K s^{2}\right]$, and $\left[K s^{3}\right]$, respectively, and $k$ and $C$ can be explicitly solved.

To demonstrate that the use of heat pulses of finite pulse duration is sufficient to recover $k$ and $C$ from the experimentally determined $T$ vs. $t$ profiles, we simulated heat transfer along a Si nanowire using COMSOL Multiphysics $^{\circledR}$ for several different types of heat inputs as shown in Fig. 2. The materials constants for a $\mathrm{Si}$ nanowire (i.e. $20 \mathrm{~nm} \times 20 \mathrm{~nm} \times 3 \mu \mathrm{m})$ were taken to be as follows: thermal conductivity $k=7 \mathrm{~W} \mathrm{~m}^{-1} \mathrm{~K}^{-1}$ (at $T_{0}=300 \mathrm{~K}$ ) [13], density $\rho=2,329 \mathrm{~kg} \mathrm{~m}^{-3}$ and specific heat 

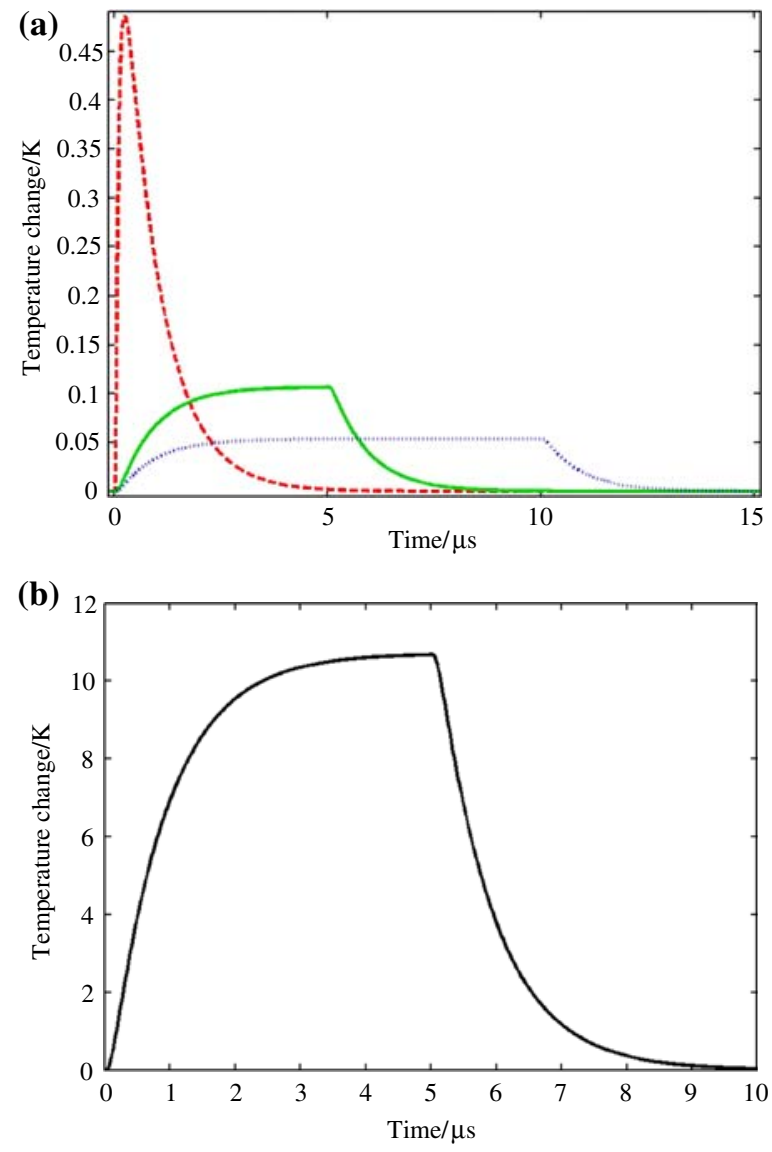

Fig. 2 a Short duration/high power ( $1 \mathrm{~ns}, 1 \mu \mathrm{W}$, dashed line), long duration/low power $(10 \mu \mathrm{s}, 100 \mathrm{pW}$, dotted line $)$, and time optimized $(5 \mu \mathrm{s}, 200 \mathrm{pW}$, solid line $)$ heat pulses responses, all at $x=1.5 \mu \mathrm{m}$ (=l/2) with $Q=1 \mathrm{fJ}$. b Time- and amplitude-optimized response from a $100 \mathrm{fJ}(5 \mu \mathrm{s}, 20 \mathrm{nW})$ heat pulse. The plots have been timeshifted (by $\sim 12 \mathrm{~ns}$ in $\mathbf{a}$, and $\sim 18 \mathrm{~ns}$ in $\mathbf{b}$ ) to omit the delay required for the excited heat wave to travel to $x=l / 2$

$C=702 \mathrm{~J} \mathrm{~kg}^{-1} \mathrm{~K}^{-1}[14]$. The initial temperature $\left(T_{0}\right)$, set to $300 \mathrm{~K}$, corresponds to a wire initially in equilibrium and in contact with a large thermal bath. An experiment in high vacuum $\left(<10^{-6}\right.$ Torr $)$ and at $300 \mathrm{~K}$ would imply thermal conduction to be the dominant heat transfer mechanism over convective and radiative effects, which have consequently been ignored.

Figure $2 \mathrm{a}$ shows the temperature response in the nanowire at the halfway point $(x=1.5 \mu \mathrm{m})$ due to three different pulse inputs, all having total energy of $1 \mathrm{fJ}: 1 \mu \mathrm{W}$ for $1 \mathrm{~ns}$ (dashed), $100 \mathrm{pW}$ for $10 \mu \mathrm{s}$ (dotted), and $200 \mathrm{pW}$ for $5 \mu$ s (solid). The $1 \mathrm{~ns}$ pulse, which excites a purely transient response, corresponds to the $\delta$-function-like pulses implicit in previous moment based methods. While the maximum temperature deviation of this profile is the largest of the three inputs $(\sim 0.5 \mathrm{~K})$, it occurs very early $(\sim 400 \mathrm{~ns})$ and has substantially decayed after several microseconds. In contrast, the $10 \mu$ s pulse introduces a more gradual heating that drives the nanowire into steady-state (for $\sim 7 \mu \mathrm{s}$ ), allowing a significantly wider time response, but produces a lower maximum temperature excursion $(\sim 50 \mathrm{mK})$. Temperature responses that have sub-Kelvin temperature changes and sub- $\mu$ s transient features impose a variety of experimental challenges such as the need for amplification hardware with extremely high speed sampling and resolution, along with rapid settling times. However, for a given pulse energy we observed through numerical simulations that such challenges could be overcome by applying an input with an optimized pulse width $\left(t_{\text {opt }}\right)$ that just achieves the onset of steady-state temperature through the wire, e.g., using a $1 \mathrm{fJ}$ pulse with a $5 \mu$ s duration. Increasing the pulse energy for the optimized duration further increases the amplitude of the temperature response. Figure $2 \mathrm{~b}$ shows the response of the $5 \mu$ s pulse with increased total energy $(100 \mathrm{fJ})$ with a temperature increment of $\sim 10 \mathrm{~K}$, which can now be easily measured. An approximate time scale for the $t_{\text {opt }}$ may also be obtained through a lumped thermal capacity model for the wire through

$t_{\mathrm{opt}} \sim \frac{L^{2} \rho C}{k}$.

For the assumed parameters, we obtain a $t_{\mathrm{opt}} \sim 2 \mu \mathrm{s}$, which is quite close to the value $(\sim 5 \mu \mathrm{s})$ determined from simulation.

Previous work using the moments method fit the $T$ vs. $t$ transient response with combinations of exponential functions [7, 8]. Generally, analytical curve fitting of rapidly varying signals requires piecewise fits and produces discontinuities, leading to inaccurate computation of numerical integrals. In our analysis, we have avoided such kind of curve fits, and instead weight the simulated temperature curve, $\Delta T(x, t)$, by $t^{n}$ to generate the integrands of Eq. 1 , for $n=0$, 1, and 2. MATLAB software was used to generate and numerically integrate linear-interpolation curve fits to yield $f_{0}, f_{1}$, and $f_{2}$. Equations $8-10$ are then solved simultaneously to determine $k$ and $C$. The values from the three possible combinations of chosen $f_{n}$, for the $1 \mathrm{~ns}$ (Fig. 2a, dashed curve) and $5 \mu$ s (Fig. 2b) pulses, are represented in Table 1.

The values for $k$ recovered from our moments analyses match very well (e.g. $2.7 \%$ for $C$ constitutes the maximum deviation) with the input parameters to within the reported significant figures. The sources of error are primarily artifacts inherent in finite element modeling, specifically: (1) finite geometrical mesh elements and, (2) finite iteration time stepping. Both sources create discretization errors over the entire $T$ vs. $t$ profile, which are further exacerbated when utilizing rapidly varying transient signals or computing higher order moments. For example, the deviations of the recovered specific heat are larger for the $1 \mathrm{~ns}$ pulse compared to the $5 \mu$ s pulse, as shown in Table 1 . Furthermore, for the $1 \mathrm{~ns}$ pulse, the error from the recovered specific heat for higher order moments $\left(2.7 \%\right.$ for $f_{1}$ and $\left.f_{2}\right)$ 
Table 1 Computed moments of simulated temperature profiles for $1 \mathrm{~ns}$ and $5 \mu$ s duration input pulses

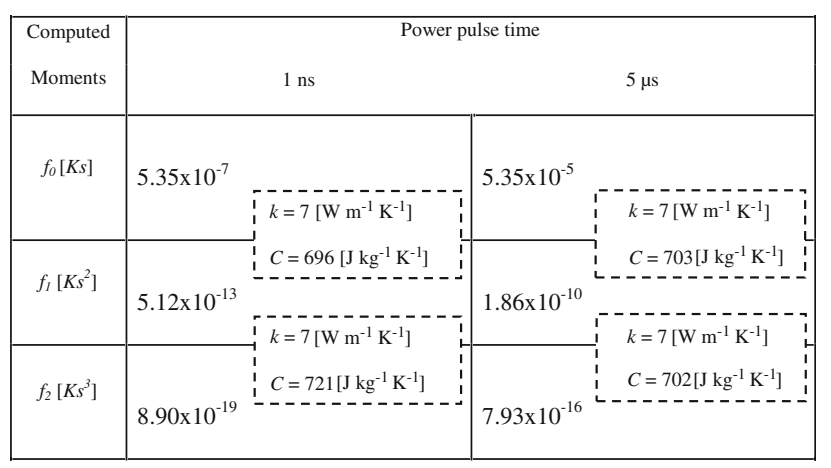

The dashed inset boxes containing the recovered thermal parameters, $k$ and $C$, are positioned to share the cells of the two moments from which they were derived

is larger than that from the lower order moments $(<1 \%$ for $f_{0}$ and $f_{1}$ ). We think that further refinement of the simulations to eliminate the above error sources is unnecessary because of their insignificance compared to intrinsic electrical noise in real experiments $[6,7]$.

A suspended nanowire similar to those previously used [15-17] can be used to demonstrate our proposed method. The nanowire would be suspended across a heating and sensing membrane to provide control of the thermal boundary conditions through: (1) Joule heating of resistive micro-heaters located near the ends of the sample, (2) thermal isolation along the length of the sample when performing the measurement in vacuum. While resting a sample across the heating and sensing membranes will impose a thermal boundary condition perpendicular to the sample axis at the points of contact, the one-dimensional approximation is still appropriate if the length $(l)$ of the wire is significantly larger than the width/height [6]. We are currently fabricating such a suspended micro-device to provide experimental proof of principle.

Our proposed method should be generally adaptable for one-dimensional nanostructures with lengths where $t_{\mathrm{opt}}$ is not too short or too long - the former would impose severe limitations on the temperature large times would enable parasitic heat transport. Other limitations of the approach involve the auxiliary effects of the electrodes and measurement apparatus which could be reduced through choosing an appropriate $t_{\mathrm{opt}}$. In conclusion, our proposed modification, through the use of finite duration heat pulses and improved computational procedure, makes more practicable the original formulation of thermal transient methods, for the determination of thermal conductivity and specific heat of nanostructures with one-dimensional heat flow.
Acknowledgements We gratefully acknowledge support from the National Science Foundation (Grant ECS-05-08514) and the Office of Naval Research (Award number N00014-06-1-0234). A. Arriagada acknowledges Mina Sierou for many helpful discussions regarding COMSOL Multiphysics ${ }^{\circledR}$ simulations.

Open Access This article is distributed under the terms of the Creative Commons Attribution Noncommercial License which permits any noncommercial use, distribution, and reproduction in any medium, provided the original author(s) and source are credited.

\section{References}

1. Carslaw HS, Jaeger JC. Conduction of heat in solids. 2nd ed. Oxford: Clarendon Press; 1959.

2. Bertman B, Heberlein DC, Sandiford DJ, Shen L, Wagner RR. Diffusive temperature pulses in solids. Cryogenics. 1970;10(4): 326-7.

3. Gershenson M, Alterovitz S. A mathematical method for the analysis of heat capacity and thermal conductivity measurement by the heat pulse technique. Appl Phys A. 1975;5(4):329-34.

4. Alterovitz S, Gershenson M. A moments method applied to laplace transform technique for experimental physics. J Comput Phys. 1975;19(2):121-33.

5. Doetsch G. Guide to the application of the Laplace and z-transforms, vol. 2. London: Van Nostrand; 1971.

6. Shapira Y, Alterovitz S. Application of a moments method and of laplace transforms to heat transfer experiments. J Therm Anal. 1980;18(3):477-91.

7. Alterovitz S, Deutscher G, Gershenson M. Heat capacity and thermal conductivity of sintered $\mathrm{Al}_{2} \mathrm{O}_{3}$ at low temperatures by the heat pulse technique. J Appl Phys. 1975;46(8):3637-43.

8. Bortner LJ, Newrock RS, Resnick DJ. An analysis of the heat pulse method for thermal transport measurements of thin films. J Appl Phys. 1987;61(9):4452-7.

9. Ghate PB. Electromigration-induced failures in VLSI interconnects. In: Proceedings of the 20th annual reliability physics symposium. New York: IEEE Press; 1982. p. 292-9

10. Filler RL, Lindenfeld P, Deutscher G. Specific heat and thermal conductivity measurements on thin films with a pulse method. Rev Sci Instrum. 1975;46(4):439-42.

11. Cruz-Uribe A, Trefny JU. The thermal properties of thin films at low temperatures using an improved heat-pulse method. J Phys E: Sci Instrum. 1982;15(10):1054-9.

12. Madsen J, Trefny JU. Boundary effects in transient thermal measurements. J Phys E: Sci Instrum. 1987;20(11):1362-5.

13. Li D, Wu Y, Kim P, Shi L, Yang P, Majumdar A. Thermal conductivity of individual silicon nanowires. Appl Phys Lett. 2003;83(14):2934-6.

14. Berger LI. Properties of semiconductors. In: Lide DR, editor. CRC handbook of chemistry and physics. 88th ed. (Internet Version 2008). Boca Raton: CRC Press/Taylor and Francis; 2008. p. 12-77.

15. Shi L, Li D, Yu C, Jang W, Kim D, Yao Z, et al. Measuring thermal and thermoelectric properties of one-dimensional nanostructures using a microfabricated device. J Heat Transf. 2003; 125(5):881-8.

16. Boukai AI, Bunimovich Y, Tahir-Kheli J, Yu JK, Goddard WA, Heath JR. Silicon nanowires as efficient thermoelectric materials. Nature. 2008;451(7175):168-71.

17. Sultan R, Avery AD, Stiehl G, Zink BL. Thermal conductivity of micromachined low-stress silicon-nitride beams from 77 to 325 K. J Appl Phys. 2009;105(4):43501-7. 